\title{
Endogenous plasma glucagon-like peptide-1 following acute dietary fibre consumption
}

\author{
Caroline L. Bodinham*, Najlaa M. Al-Mana, Leanne Smith and M. Denise Robertson \\ Department of Nutritional Sciences, University of Surrey, Leggett Building, Guildford, Surrey GU2 7WG, UK
}

(Submitted 30 May 2012 - Final revision received 28 January 2013 - Accepted 28 January 2013 - First published online 18 March 2013)

\section{Abstract}

SCFA resulting from the microbial fermentation of carbohydrates have been linked to increased glucagon-like peptide-1 (GLP-1) secretion from the gastrointestinal tract in cell and animal models; however, there is little direct evidence in human subjects to confirm this. The present study was designed to investigate whether endogenous plasma GLP-1 concentrations increase following acute consumption of $48 \mathrm{~g}$ dietary fibre (as resistant starch (RS) from high-amylose maize type 2 RS (HAM-RS2)) compared with a matched placebo. A total of thirty healthy males participated in the present randomised cross-over study where HAM-RS2 or placebo was consumed as part of standardised breakfast and lunch meals. Changes to GLP-1, glucose, insulin and C-peptide were assessed half hourly for $7 \mathrm{~h}$. Following the breakfast meal, plasma GLP-1 concentrations were lower with HAM-RS2 compared with the placebo $(P=0 \cdot 025)$. However, there was no significant difference between the supplements following the lunch meal. Plasma insulin concentrations were significantly lower following the lunch meal $(P=0.034)$ with HAM-RS2 than with the placebo, but were not different after breakfast. Plasma glucose and C-peptide concentrations did not differ at any point. These results suggest that increased dietary fibre intake, in the form of HAM-RS2, does not acutely increase endogenous GLP-1 concentrations in human subjects. Further fibre feeding studies are required to determine whether GLP-1 concentrations may increase following longer-term consumption.

\section{Key words: Resistant starch: Dietary fibre: Glucagon-like peptide-1}

Glucagon-like peptide-1 (GLP-1) is secreted from the endocrine $\mathrm{L}$ cells in the small and large intestine. GLP- $1_{7-36}$ (the most biologically active form) is rapidly broken down by protease enzymes, such as dipeptidyl peptidase-IV, to GLP-1 ${ }_{9-36}$. GLP-1 has been well characterised as having two main roles as a satiety hormone and as an incretin. As a satiety hormone, GLP-1 has been shown to both enhance satiety and reduce food intake, and has been proposed to be useful as a treatment for weight management. The role of GLP-1 as an incretin has been well defined, and it is believed that the action of incretins may account for at least $50 \%$ of insulin that is released in response to oral glucose ${ }^{(1)}$. As a result, analogues of GLP-1 ${ }_{7-36}$, which are resistant to dipeptidyl peptidase-IV, are being used as a therapeutic treatment for type 2 diabetes.

Dietary carbohydrates that pass through the small intestine to the colon can be classified as dietary fibre; these can act as a substrate for the gut microbiota, leading to the production of SCFA. It is these bioactive SCFA that in turn have been proposed to increase the production of GLP-1. This is thought to be mediated through SCFA binding to G-proteincoupled receptors (free fatty acid receptor $2 / 3$; FFAR2/3) on
GLP-1-secreting L cells in the colon ${ }^{(2)}$. Therefore, the products of the fermentation of fibres may cause an increase in endogenous GLP-1 concentrations and thus may potentiate the effects of GLP-1 as an incretin as well as a satiety signal.

Resistant starch (RS), a non-viscous fermentable dietary fibre, has been shown in many animal studies to increase GLP-1 production ${ }^{(3-5)}$. Studies in human subjects where GLP-1 has been measured following increased RS feeding are limited ${ }^{(6-8)}$, with only one of these being an acute feeding study ${ }^{(8)}$, and these have predominately shown no effect of RS on GLP-1 concentrations. However, the beneficial effects that have been observed following increased RS consumption, ranging from $24 \mathrm{~h}$ to 12 weeks, have been an improvement in insulin sensitivity ${ }^{(6,7,9,10)}$, insulin secretion ${ }^{(11)}$, a reduction in food intake ${ }^{(12)}$ and increased satiety ${ }^{(13,14)}$. The only potential candidate that could fulfil all of these roles would be GLP-1.

Our group has previously demonstrated a significant reduction in food intake following increased RS intakes (from high-amylose maize type 2 RS (HAM-RS2)) ${ }^{(12)}$. The present study therefore aimed to follow a similar protocol but specifically measuring the endogenous secretion of GLP-1 and postprandial insulin and glucose concentrations.

Abbreviations: GLP-1, glucagon-like peptide-1; HAM-RS2, high-amylose maize type 2 resistant starch; iAUC, incremental AUC; RS, resistant starch.

*Corresponding author: Dr C. L. Bodinham, fax + 441483 688501, email caroline.bodinham@surrey.ac.uk 
The study aimed to determine whether the previously observed reductions in food intake and changes to postprandial insulin concentrations could be due to increases in endogenous GLP-1 concentrations.

\section{Experimental methods}

\section{Participants}

A total of thirty healthy adult males, with a mean age of 24.5 (SEm 0.7) years and a mean BMI of $26 \cdot 2$ (SEm 0.9) kg/m², participated in the present study. Participants were excluded if they had a history of gastrointestinal or endocrine disease. The present study was conducted according to the guidelines laid down in the Declaration of Helsinki, and all procedures involving human subjects were approved by the University of Surrey Ethics Committee. Written informed consent was obtained from all participants.

\section{Study design}

The study design was based on that described previously ${ }^{(12)}$. The study was a single-blind, cross-over study in which participants visited the clinical unit on two occasions at least 1 week apart. On these clinical visits, participants consumed either $80 \mathrm{~g}$ Hi-Maize $260^{\circledR}$ (comprising 60\% type 2 RS (HAM-RS2) and 40\% rapidly digestible starch (RDS), therefore providing $48 \mathrm{~g}$ HAM-RS2 as measured by the Association of Official Analytical Chemists for total dietary fibre method 991.43) or $32 \mathrm{~g}$ of placebo (Amioca ${ }^{\circledR}$, comprising 100\% RDS) supplying no dietary fibre. Both starches were manufactured and supplied by Ingredion Inc. Half of the starch was provided at breakfast and the other half at lunch, incorporated into a mousse, therefore providing $24 \mathrm{~g}$ dietary fibre or $0 \mathrm{~g}$ dietary fibre at each meal.

On arrival at the clinical unit, a cannula was placed into the antecubital fossa and two fasted blood samples were taken. At time zero, participants consumed the standardised breakfast ( $1587 \mathrm{~kJ}, 9.6 \mathrm{~g}$ protein, $67 \cdot 1 \mathrm{~g}$ carbohydrate, $8.0 \mathrm{~g}$ fat and $0.5 \mathrm{~g}$ dietary fibre (24.5 g dietary fibre for the HAM-RS2 supplement)). Following the breakfast, blood samples were taken every $30 \mathrm{~min}$ for $3 \mathrm{~h}$. Participants were then provided with the standardised lunch $(3888 \mathrm{~kJ}, 36 \cdot 1 \mathrm{~g}$ protein, $112 \cdot 1 \mathrm{~g}$ carbohydrate, $37 \cdot 0 \mathrm{~g}$ fat and $5.0 \mathrm{~g}$ dietary fibre (29.0 g dietary fibre for the HAM-RS2 supplement)). Further blood samples were collected for the following $4 \mathrm{~h}$. Bowel habit and symptom diaries were completed on the day of the study and the following day to assess gastrointestinal tolerance of the starches.

\section{Biochemistry}

Whole blood was collected into fluoroxalate tubes for glucose analysis, potassium EDTA tubes for insulin analysis and potassium EDTA tubes with 200 kallikrein-inhibiting units aprotinin per $\mathrm{ml}$ of blood for C-peptide and total GLP-1 concentrations. All samples were centrifuged for $10 \mathrm{~min}$ at $3000 \mathrm{rpm}$ and plasma stored at $-20^{\circ} \mathrm{C}$ until batch analysis. Plasma glucose concentrations were measured enzymatically using a commercially available kit for the ILab650 (Instrumentation Laboratory) and inter-assay variation was $<2 \%$. Plasma insulin and C-peptide concentrations were measured by RIA using commercially available kits (Millipore) with inter-assay variations of 18.2 and $17 \cdot 7 \%$, respectively, and intra-assay variations of 9.2 and $9.5 \%$, respectively. Plasma total GLP-1 concentrations were measured by ELISA using a commercially available kit (Millipore) with an inter-assay variation of $15.0 \%$ and an intra-assay variation of $4.5 \%$.

\section{A priori power calculation and statistics}

The thirty participants in the present cross-over study ( $\alpha=0.05$ ) would yield an $80 \%$ probability of detecting a $540 \mathrm{pmol} / 1$ per $420 \mathrm{~min}$ change in postprandial GLP-1 response (AUC, based on a treatment SD of 1018). All postprandial time course data were analysed using repeatedmeasures ANOVA. The incremental AUC (iAUC) was calculated for each meal using the trapezoid method, and then the iAUC was compared between the treatments using

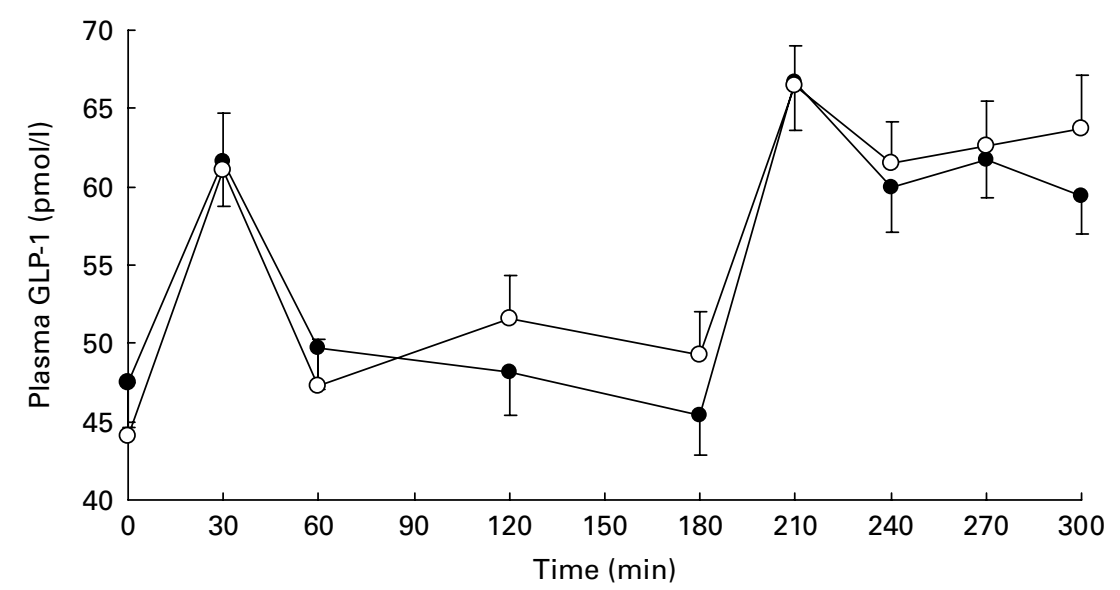

Fig. 1. Postprandial plasma glucagon-like peptide-1 (GLP-1) concentrations. Values are means for thirty participants following $48 \mathrm{~g}$ high-amylose maize type 2 resistant starch $(\bullet)$ or placebo $(O)$ consumption; consumed in two equal portions, one at time zero and the other at 180 min, with their standard errors represented by vertical bars. There was a significant treatment $\times$ time interaction using repeated-measures ANOVA $(P=0.020)$. 
paired $t$ tests. All statistical analyses were carried out using SPSS version 18 for Windows (SPSS, Inc.) with significance assumed as $P<0.05$. All results presented are means with their standard errors.

\section{Results}

Both HAM-RS2 and placebo were well tolerated by the participants with a similar frequency of bowel movements recorded and no adverse gastrointestinal effects reported on the day of the study or the following day. Participants' weight did not differ between the two study days.

\section{Glucagon-like peptide-1 concentrations}

There was a significant treatment $\times$ time interaction between HAM-RS2 and placebo for postprandial GLP-1 concentrations $(P=0 \cdot 020$; Fig. 1).

The iAUC for each meal showed a significantly lower GLP-1 response with HAM-RS2 compared with placebo following breakfast $(P=0.025$; Fig. 2(a)). However, following the lunch meal, there were no differences between the starches (Fig. 2(a)).
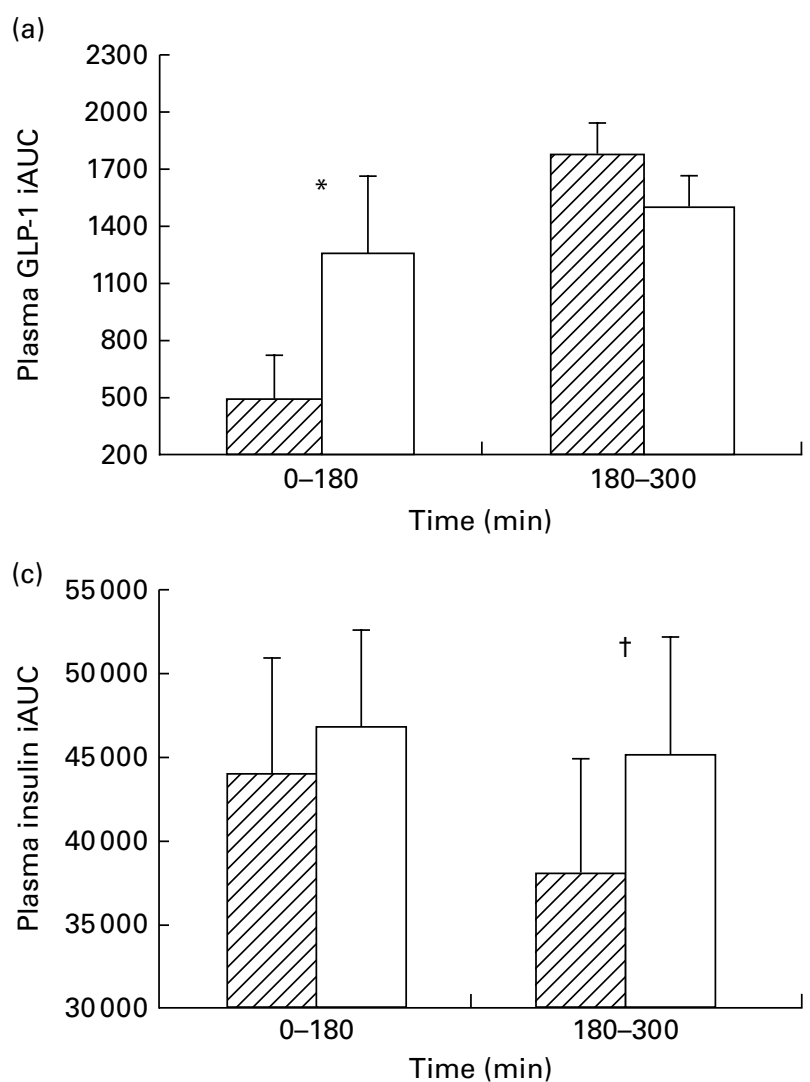

\section{Postprandial metabolite concentrations}

Over the whole $7 \mathrm{~h}$ postprandial period, there were no differences between HAM-RS2 and placebo for concentrations of plasma glucose, insulin or C-peptide.

The iAUC for insulin following breakfast $(0-180 \mathrm{~min})$ were not different between HAM-RS2 and placebo; however, after lunch (180-300 $\mathrm{min}$ ), there was a significantly lower response following HAM-RS2 than with placebo $(P=0 \cdot 034$; Fig. $2(\mathrm{c}))$.

There were no significant differences in iAUC between the RS and placebo for glucose or C-peptide (Fig. 2(b) and (d), respectively).

\section{Discussion}

To our knowledge, this is the first study that has investigated the effects of increased HAM-RS2 intake on endogenous GLP-1 concentrations when compared with a placebo matched for both energy and glycaemic load, in human subjects. The present study has shown that during the $3 \mathrm{~h}$ following a breakfast meal containing HAM-RS2, there was a significant reduction in GLP-1 concentrations compared with placebo. However, after a further HAM-RS2-containing meal, there was no difference in GLP-1 concentrations between the supplements.
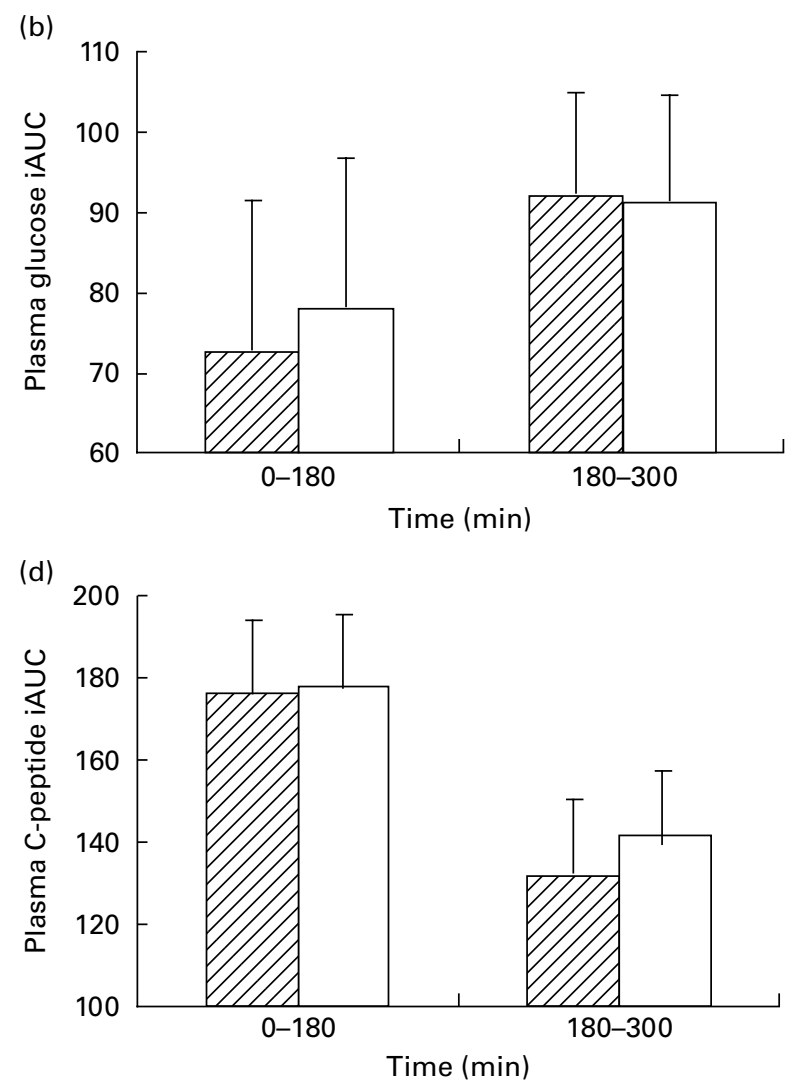

Fig. 2. Incremental AUC (iAUC) at each meal for plasma (a) glucagon-like peptide-1 (GLP-1), (b) glucose, (c) insulin and (d) C-peptide. Values are means for thirty participants following $48 \mathrm{~g}$ high-amylose maize type 2 resistant starch (HAM-RS2, $\square$ ) or placebo ( $\square$ ) consumption; consumed in two equal portions at breakfast (time zero) and lunch $(180 \mathrm{~min})$, with their standard errors represented by vertical bars. * Mean value was significantly different for GLP-1 iAUC at time 0-180 min with HAM-RS2 compared with placebo $(P=0.025)$ but not significantly different at time $180-300$ min. $†$ Mean value was significantly different for insulin iAUC at time $180-300$ min with HAM-RS2 compared with placebo $(P=0.034)$ but not significantly different at time $0-180$ min. Mean values were not significantly different for glucose or C-peptide concentrations. 
In the present acute postprandial study, increased intakes of $48 \mathrm{~g}$ HAM-RS2, a non-viscous fermentable fibre, did not increase plasma GLP-1 concentrations and, indeed, following the breakfast meal, GLP-1 concentrations were significantly lower with the RS than compared with the placebo. There is little previous direct evidence for an effect of RS on GLP-1 in human subjects. A postprandial study comparing $27 \mathrm{~g} \mathrm{RS}$ (from raw potato starch) with a placebo (that was not matched for glycaemic carbohydrate) also found lower GLP-1 with the $\mathrm{RS}^{(8)}$. In the present study, there was a significant treatment $\times$ time interaction, translating as the RS-containing meal giving a lower acute GLP-1 response with higher post-lunch levels, when compared with placebo (Fig. 1). This may suggest that the increase in GLP-1 concentrations was due to the fermentation of HAM-RS2, and investigating the effects later on in the day or even the subsequent morning may have produced significant differences in GLP-1 concentrations. However, one study in which $60 \mathrm{~g}$ HAM-RS2 was consumed for $24 \mathrm{~h}$ before the investigation compared with the same placebo found no effect of HAM-RS2 on GLP-1 concentrations $^{(6)}$. Another study looking at carry-over effects of evening meals varying in indigestible carbohydrate content found no effect of evening meals on fasting GLP-1 concentrations the following day, but did demonstrate a significantly higher GLP-1 AUC (0-120 min) following one treatment (barley kernels containing $20 \cdot 2 \mathrm{~g}$ of total dietary fibre) compared with the control product $(3.9 \mathrm{~g}$ of total dietary fibre $)^{(15)}$. Additionally, a study in which $30 \mathrm{~g}$ HAM-RS2 was consumed daily for 4 weeks compared with a matched glycaemic carbohydrate placebo again found no effect of HAM-RS2 on GLP-1 ${ }^{(7)}$, although, unlike the present study, this was not an acute response to the fibre. Overall, the data from the present study and from the previous studies would suggest that HAM-RS2 does not cause an increase in endogenous GLP-1, particularly when HAM-RS2 is consumed acutely.

While the GLP-1 data in human subjects following increased RS intake are limited, there is much animal work that has shown consistent increases in GLP-1 blood concentration following higher levels of RS feeding ${ }^{(3-5)}$ as well as increases in proglucagon gene expression in the caecum and colon ${ }^{(3,4,16)}$. However, it is difficult to directly translate these findings as the studies in rodents have not been acute (days rather than hours), and therefore it may be that prolonged exposure to HAM-RS2 in human subjects is required to affect GLP-1 concentrations. A previous study in human subjects has shown an increase in GLP-1 concentrations following wheat fibre intake, although a year of increased wheat fibre feeding was required before the effect was observed ${ }^{(17)}$

Other acute studies using increased fibre feeding have shown inconsistent results. A $2 \mathrm{~h}$ postprandial study feeding a galactose and guar gum-mixed drink compared with a control drink found increased GLP-1 concentrations ${ }^{(18)}$; another $6 \mathrm{~h}$ postprandial study comparing an inulin drink with highfructose maize syrup drinks found GLP-1 concentrations to be higher with inulin at $30 \mathrm{~min}^{(19)}$. However, other studies where dietary fibres have been given acutely, as in the present study, have found no effect on GLP-1 concentrations ${ }^{(20,21)}$. Overall, a recent review has concluded that the current research on the effects of fibres on GLP-1 demonstrates that fibres do not increase GLP-1 concentrations compared with control; however, the authors have acknowledged that there are few studies that have actually assessed the effects of fibre on gut hormones directly ${ }^{(22)}$.

Previously, we have shown that acute ingestion of HAM-RS2 reduced postprandial insulin concentrations ${ }^{(12)}$, which we have demonstrated again in the present study following the second dose of HAM-RS2, but not following the first dose, or over the whole $7 \mathrm{~h}$ period. In the present study, the lower insulin response observed after the second dose was found in conjunction with a higher GLP-1 concentration, which is not in agreement with GLP-1 acting as an incretin following fibre feeding. This would therefore suggest that following increased RS intakes, GLP-1 is unlikely to have a role as an incretin. In many experimental designs, RS has replaced portions of digestible starch, or ratios of amylose: amylopectin have been manipulated (changing glycaemic index and/or glycaemic load), thus making comparisons problematic. A recent review of the literature related to RS and glycaemia/insulinaemia highlighted this disparity ${ }^{(23)}$. In our previous work, we hypothesised that a reduced postprandial insulin response often attributed to RS intake may be due to increased hepatic insulin clearance ${ }^{(12)}$; however, we found no evidence to support this in the present study.

The limitations of the present study are that we only measured total as opposed to active GLP- $1_{7-36}$ concentrations and therefore the effects of GLP-1 as a satiety signal may be masked. As recently reviewed, gut hormones may not act independently, and it may be the additive effects of several gut hormones that result in observed changes to satiety ${ }^{(22)}$. Indeed, it is established that the postprandial levels of GLP-1 attained during feeding would have minimal effects on food intake ${ }^{(24,25)}$, when considered in isolation. However, GLP-19-36 may still have a role as an incretin, as some studies have suggested that it may reduce glycaemia to a small degree independently to any effects on insulin secretion ${ }^{(26,27)}$. Therefore, the relevance of measuring total GLP-1 concentrations needs to be considered in an attempt to understand the health benefits of dietary fibre intake.

Overall, it would appear from the present study and that of the literature that GLP-1 is not a candidate signal for the acutely reduced food intake and enhanced insulin sensitivity previously attributed to HAM-RS2.

\section{Acknowledgements}

We thank all of the participants for their time. The present study was funded by Ingredion Inc. but the industrial funder had no involvement in the study design or data analysis and interpretation. N. M. A.-M. was funded by an Educational Scholarship from the Saudi Arabian Government, King Saud University for Health Science. The supplements were supplied by Ingredion Inc. C. L. B. was involved in the designing of the study protocol, recruitment of the volunteers, conducting of the study days, analysis of the data, statistical analysis and writing of the paper. N. M. A.-M. was involved in the recruitment of the volunteers, and the conducting of the study days 
and analysis of the data. L. S. was involved in the recruitment of the volunteers, and the conducting of the study days and analysis of the data. M. D. R. was involved in the designing of the study protocol, assistance on the study days, analysis of the data, statistical analysis and refining of the paper.

\section{References}

1. Kim W \& Egan JM (2008) The role of incretins in glucose homeostasis and diabetes treatment. Pharmacol Rev 60 , $470-512$.

2. Tolhurst G, Heffron H, Lam YS, et al. (2012) Short-chain fatty acids stimulate glucagon-like peptide-1 secretion via the G-protein-coupled receptor FFAR2. Diabetes 61, 364-371.

3. Zhou J, Martin RJ, Tulley RT, et al. (2008) Dietary resistant starch upregulates total GLP-1 and PYY in a sustained daylong manner through fermentation in rodents. Am J Physiol Endocrinol Metab 295, E1160-E1166.

4. Keenan MJ, Zhou J, McCutcheon KL, et al. (2006) Effects of resistant starch, a non-digestible fermentable fiber, on reducing body fat. Obesity (Silver Spring) 14, 1523-1534.

5. Shen L, Keenan MJ, Martin RJ, et al. (2009) Dietary resistant starch increases hypothalamic POMC expression in rats. Obesity (Silver Spring) 17, 40-45.

6. Robertson MD, Currie JM, Morgan LM, et al. (2003) Prior short-term consumption of resistant starch enhances postprandial insulin sensitivity in healthy subjects. Diabetologia 46, 659-665.

7. Robertson MD, Bickerton AS, Dennis AL, et al. (2005) Insulin-sensitizing effects of dietary resistant starch and effects on skeletal muscle and adipose tissue metabolism. Am J Clin Nutr 82, 559-567.

8. Raben A, Tagliabue A, Christensen NJ, et al. (1994) Resistant starch: the effect on postprandial glycemia, hormonal response, and satiety. Am J Clin Nutr 60, 544-551.

9. Johnston KL, Thomas EL, Bell JD, et al. (2010) Resistant starch improves insulin sensitivity in metabolic syndrome. Diabet Med 27, 391-397.

10. Maki KC, Pelkman CL, Finocchiaro ET, et al. (2012) Resistant starch from high-amylose maize increases insulin sensitivity in overweight and obese men. J Nutr 142, 717-723.

11. Bodinham CL, Smith L, Wright J, et al. (2012) Dietary fibre improves first-phase insulin secretion in overweight individuals. PLoS One 7, e40834.

12. Bodinham CL, Frost GS \& Robertson MD (2010) Acute ingestion of resistant starch reduces food intake in healthy adults. Br J Nutr 103, 917-922.

13. Willis HJ, Eldridge AL, Beiseigel J, et al. (2009) Greater satiety response with resistant starch and corn bran in human subjects. Nutr Res 29, 100-105.
14. Liljeberg HG, Akerberg AK \& Bjorck IM (1999) Effect of the glycemic index and content of indigestible carbohydrates of cereal-based breakfast meals on glucose tolerance at lunch in healthy subjects. Am J Clin Nutr 69, 647-655.

15. Nilsson AC, Ostman EM, Holst JJ, et al. (2008) Including indigestible carbohydrates in the evening meal of healthy subjects improves glucose tolerance, lowers inflammatory markers, and increases satiety after a subsequent standardized breakfast. J Nutr 138, 732-739.

16. Zhou J, Hegsted M, McCutcheon KL, et al. (2006) Peptide YY and proglucagon mRNA expression patterns and regulation in the gut. Obesity (Silver Spring) 14, 683-689.

17. Freeland KR, Wilson C \& Wolever TM (2010) Adaptation of colonic fermentation and glucagon-like peptide-1 secretion with increased wheat fibre intake for 1 year in hyperinsulinaemic human subjects. Br J Nutr 103, 82-90.

18. Adam TC \& Westerterp-Plantenga MS (2005) Glucagon-like peptide- 1 release and satiety after a nutrient challenge in normal-weight and obese subjects. Br J Nutr 93, 845-851.

19. Tarini J \& Wolever TM (2010) The fermentable fibre inulin increases postprandial serum short-chain fatty acids and reduces free-fatty acids and ghrelin in healthy subjects. Appl Physiol Nutr Metab 35, 9-16.

20. Barone Lumaga R, Azzali D, Fogliano V, et al. (2012) Sugar and dietary fibre composition influence, by different hormonal response, the satiating capacity of a fruit-based and a beta-glucan-enriched beverage. Food Funct 3, 67-75.

21. Frost GS, Brynes AE, Dhillo WS, et al. (2003) The effects of fiber enrichment of pasta and fat content on gastric emptying, GLP-1, glucose, and insulin responses to a meal. Eur J Clin Nutr 57, 293-298.

22. Klosterbuer A, Greaves K \& Slavin J (2012) Fiber intake inconsistently alters gut hormone levels in humans following acute or chronic intake. J Food Res 1, 255-273.

23. Robertson MD (2012) Dietary-resistant starch and glucose metabolism. Curr Opin Clin Nutr Metab Care 15, 362-367.

24. Flint A, Raben A, Ersboll AK, et al. (2001) The effect of physiological levels of glucagon-like peptide-1 on appetite, gastric emptying, energy and substrate metabolism in obesity. Int J Obes Relat Metab Disord 25, 781-792.

25. Verdich C, Toubro S, Buemann B, et al. (2001) The role of postprandial releases of insulin and incretin hormones in meal-induced satiety - effect of obesity and weight reduction. Int J Obes Relat Metab Disord 25, 1206-1214.

26. Elahi D, Egan JM, Shannon RP, et al. (2008) GLP-1 (9-36) amide, cleavage product of GLP-1 (7-36) amide, is a glucoregulatory peptide. Obesity (Silver Spring) 16, 1501-1509.

27. Meier JJ, Gethmann A, Nauck MA, et al. (2006) The glucagon-like peptide-1 metabolite GLP-1-(9-36) amide reduces postprandial glycemia independently of gastric emptying and insulin secretion in humans. Am J Physiol Endocrinol Metab 290, E1118-E1123. 